\title{
High Sensitivity Refractive Index Sensor Based on a Tapered Small Core Single-Mode Fiber Structure.
}

\author{
Dejun Liu \\ Technological University Dublin, dejun.liu@tudublin.ie \\ Arun Mallik \\ Technological University Dublin, arun.mallik@tudublin.ie \\ Jinhui Yuan \\ Department of Physics and Electrical Engineering, Northumbria University, UK
}

See next page for additional authors

Follow this and additional works at: https://arrow.tudublin.ie/engschmanart

Part of the Engineering Commons

\section{Recommended Citation \\ This Article is brought to you for free and open access by the School of Manufacturing and Design Engineering at ARROW@TU Dublin. It has been accepted for inclusion in Articles by an authorized administrator of ARROW@TU Dublin. For more information, please contact arrow.admin@tudublin.ie, aisling.coyne@tudublin.ie, gerard.connolly@tudublin.ie.}

Mallik, A. (2015). High sensitivity refractive index sensor based on a tepered small core single-mode fiber structure. Optics Letters, vol. 40, no. 17, pp. 4166-4169. doi:10.1364/OL.40.004166.

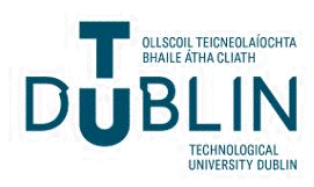




\section{Authors}

Dejun Liu, Arun Mallik, Jinhui Yuan, Chongxiu Yu, Gerald Farrell, Yuliya Semenova, and Qiang wu 


\title{
High sensitivity refractive index sensor based on a tapered small core single-mode fiber structure
}

\author{
Dejun Liu, ${ }^{1}$ Arun Kumar Mallik, ${ }^{1}$ Jinhui Yuan, ${ }^{3}$ Chongxiu Yu, ${ }^{3}$ Gerald Farrell, ${ }^{1}$ \\ Yuliya Semenova, ${ }^{1}$ and Qiang Wu $\mathbf{u}^{1,2,3, *}$ \\ ${ }^{1}$ Photonics Research Center, Dublin Institute of Technology, Kevin Street, Dublin 8, Ireland \\ ${ }^{2}$ Department of Physics and Electrical Engineering, Northumbria University, Newcastle Upon Tyne, NE1 8ST, UK \\ ${ }^{3}$ State Key Laboratory of Information Photonics and Optical Communications, Beijing University of Posts and Telecommunications, \\ Beijing 100876, China \\ *Corresponding author: qiang.wu@northumbria.ac.uk
}

Received 15 June 2015; revised 6 August 2015; accepted 11 August 2015; posted 11 August 2015 (Doc. ID 242978); published 1 September 2015

\begin{abstract}
A high sensitivity refractive index (RI) sensor based on a tapered small core single-mode fiber (SCSMF) structure sandwiched between two traditional single-mode fibers (SMF28) is reported. The microheater brushing technique was employed to fabricate the tapered fiber structures with different waist diameters of $12.5,15.0$, and $18.8 \mu \mathrm{m}$. Experiments demonstrate that the fiber sensor with a waist diameter of $12.5 \mu \mathrm{m}$ offers the best sensitivity of $19212.5 \mathrm{~nm} / \mathrm{RIU}$ (RI unit) in the RI range of 1.4304 to 1.4320. All sensors fabricated in this Letter show good linearity in terms of the spectral wavelength shift versus changes in RI. Furthermore, the sensor with the best sensitivity to RI was also used to measure relative humidity (RH) without any coating materials applied to the fiber surface. Experimental results show that the spectral wavelength shift changes exponentially as the $\mathrm{RH}$ varies from $60 \%$ to $95 \%$. A maximum sensitivity of $18.3 \mathrm{~nm}$ per relative humidity unit (RHU) was achieved in the $\mathrm{RH}$ range of $90.4 \%$ to 94.5\% RH. @ 2015 Optical Society of America
\end{abstract}

OCIS codes: (060.2280) Fiber design and fabrication; (060.2310) Fiber optics; (060.2370) Fiber optics sensors; (060.4005) Microstructured fibers.

http://dx.doi.org/10.1364/OL.40.004166

Optical fiber sensors show great potential for applications involving measurements of gas and liquid concentrations, compound materials solidification monitoring, and biomolecules detection, where sensing of the surrounding refractive index (SRI) is used as an underlying principle [1,2]. To date, a number of approaches have been proposed for refractive index (RI) sensors, including fiber Bragg gratings [3], surface plasmon resonance [4], single-mode-multimode-single-mode (SMS) fiber structures [5], optical ring resonators [6], and optical microfiber couplers [7]. Among these structures, SMS-fiber-structurebased sensors are attractive, given the additional advantages of low cost and ease of fabrication. However, to fabricate an SMS-based RI sensor, a chemical etching process is required to remove the cladding from the multimode fiber (MMF) section, which may introduce some problems with surface roughness of the etched fiber, in addition to safety concerns [5].

A small core single-mode fiber (SCSMF) is a good candidate to replace the MMF section in an SMS fiber structure, with the advantage that etching is not required. In our previous work, we have proved theoretically and experimentally that a SCSMF-based fiber structure can act as a high sensitivity RI sensor with a maximum sensitivity of $1808 \mathrm{~nm} / \mathrm{RIU}$ [8]. Furthermore, we have demonstrated as an application of the structure, a humidity sensor utilizing a coating with a maximum sensitivity of $430 \mathrm{~nm} / \mathrm{RHU}$ [9]. Such sensitivities are still inadequate in some applications, particularly in bio-sensing, which requires detection of extremely small RI variations. Recently, microfiber and nanofiber sensors have attracted significant interest as they offer a range of advantages, such as large evanescent fields, high nonlinearity, low loss connections to standard fibers, and strong mode confinement [10]. For example, Yadav et al. reported an RI sensor with a sensitivity of $1500 \mathrm{~nm} / \mathrm{RIU}$ based on a tapered single-mode fiber [11]. Most recently, Zhu et al. developed a highly sensitive RI sensor with a sensitivity of $6008 \mathrm{~nm} / \mathrm{RIU}$ by coating a $\mathrm{Al}_{2} \mathrm{O}_{3}$ nanofilm on a tapered fiber [12]. Xu et al. have demonstrated an RI sensor with a sensitivity of $6523 \mathrm{~nm} /$ RIU based on cascaded microfiber knot resonators (CMKRs) with Vernier effect [13].

In this Letter, following on our previous work, we report that by tapering the SCSMF structure, an improved RI sensor is possible with a sensitivity that is an order of magnitude higher than the untapered device we reported in [8]. The maximum demonstrated sensitivity was estimated as $19212.5 \mathrm{~nm} / \mathrm{RIU}$ in the RI range of 1.4304-1.4320. All the sensor samples studied in this Letter show good linearity of the wavelength shift versus surrounding RI change. Furthermore, one of the sensors with the highest RI sensitivity is also chosen to show that the tangible benefit of the much improved RI sensitivity is that we show it is possible to implement a relative humidity $(\mathrm{RH})$ sensor without the need for any additional humidity sensitive coatings on the fiber surface.

In a typical SMF28-SCSMF-SMF28 fiber structure, cladding modes are excited within the cladding of the SCSMF 
section because of the core diameter mismatch between SMF28 and SCSMF. Multimode interference for these cladding modes occurs within the SCSMF section. It is well known that the propagation constant of the cladding mode (corresponding to effective RI) is influenced by the RI of the surrounding environment. Therefore, a change in the SRI for the sensor will affect the multimode interference, and, in turn, this will result in changes in the spectral response which can be monitored by an optical spectrum analyzer (OSA). Thus, the SRI can be measured by monitoring the variations of the spectral response, assuming a suitable calibration has taken place. This type of sensor is often referred to as an "evanescent sensor."

Typically an increase in the portion of the evanescent field exposed to the surrounding environment results in a higher sensitivity for the sensor [10]. A simple, non-tapered SCSMF structure has a relatively large diameter (typically $125 \mu \mathrm{m}$ ), resulting in a relatively small portion of the evanescent field being in contact with the surrounding environment, and, hence, such a sensor has relatively low sensitivity. One of the solutions to improve the sensitivity is to use a tapered SCSMF which has a smaller waist diameter, as reported in our previous work [14]. In this Letter, we have systematically investigated the tapered SCSMF structure for RI sensing, where the diameters of the SCSMF are tapered down to $12.5,15.0$, and $18.8 \mu \mathrm{m}$.

In our experiments, a section of SCSMF (SM450) with a length of circa $22.5 \mathrm{~mm}$ was fusion spliced between two SMF-28 fibers. The SCSMF section was then tapered using a microheater brushing technique as shown in Fig. 1(a) [15]. Three SCSMF sensor samples were fabricated with different tapered waist diameters $(18.8,15.0$, and $12.5 \mu \mathrm{m})$. The waist diameter was measured by a microscope. The prepared sensor samples are referred hereinafter to as S-18.8, S-15.0, and S-12.5. For each taper, the taper waist length is the same with a length of approximately $3.5 \mathrm{~mm}$. The taper transition lengths for S-18.8, S-15.0, and S-12.5 are circa $8 \mathrm{~mm}, 8.5 \mathrm{~mm}$, and $9 \mathrm{~mm}$, respectively. In each case the tapered SCSMF structure was fixed on a glass slide ensuring that the fiber sensor was always straight and the sensing section was slightly above the slide to avoid any physical contact with the glass surface.

Figure 1(b) illustrates a schematic diagram of the RI measuring system and a tapered fiber structure, also showing a microscopic image of the taper waist for S-12.5. Light from a broadband light source (Thorlabs S5FC1005S) 1450-1650 nm is launched into the tapered SCSMF structure, while the transmitted light is measured by an OSA (Agilent 86142B). The RI liquid can be placed on the glass slide so that the tapered SCSMF fiber section is immersed in the liquid. All tests were conducted at room temperature.

Figure 2 shows three examples of the measured spectral responses for the S-12.5 sensor immersed in various liquids with calibrated RI values. The RI value ranges are (a) 1.34051.3463 , (b) $1.3748-1.3837$, and (c) 1.4304-1.4311. The spectral dip moves monotonically toward longer wavelengths as the SRI increases in every case. The S-18.8 and S-15.0 sensors have different spectral responses, but the direction of the wavelength shift with increasing SRI is the same. The spectral responses for the S-18.8 and S-15.0 sensors are not shown here for the sake of brevity.

The dip wavelength shifts versus RI change for all the sensors are presented in Fig. 3, and summarized in detail in Table 1. As expected, RI sensitivity increases as the tapered (a)

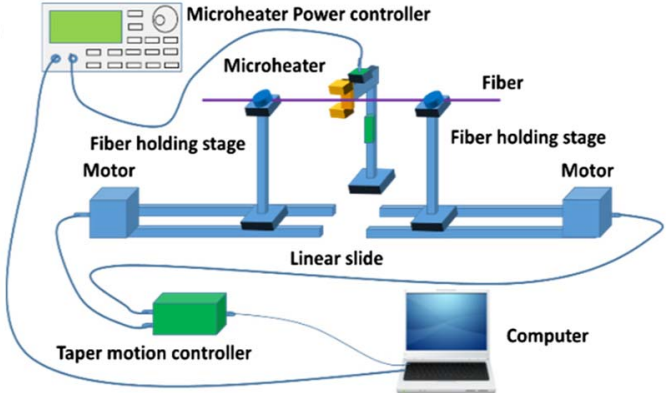

(b)

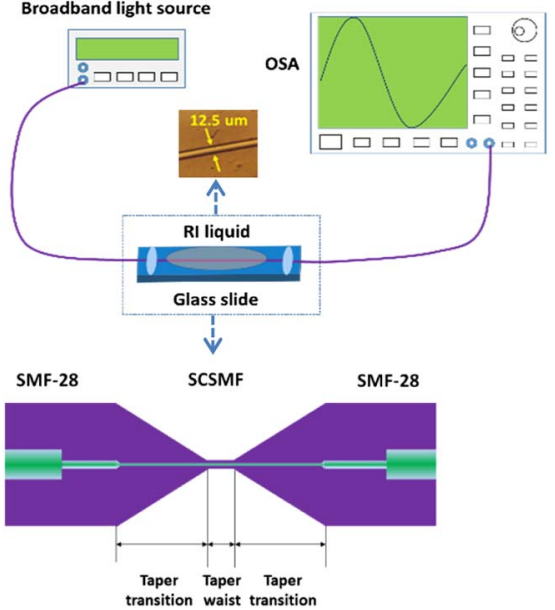

Fig. 1. Schematic diagram of (a) the microfiber tapering setup and (b) the RI sensing system setup.

waist diameter decreases. This is likely because of the fact that smaller waist diameter sensors have a larger portion of the evanescent field exposed to the surrounding environment. Figure 3 also shows that in the higher RI range, all three samples have higher sensitivity. The likely explanation for this is that, as light propagates in the fiber, it experiences a larger penetration depth

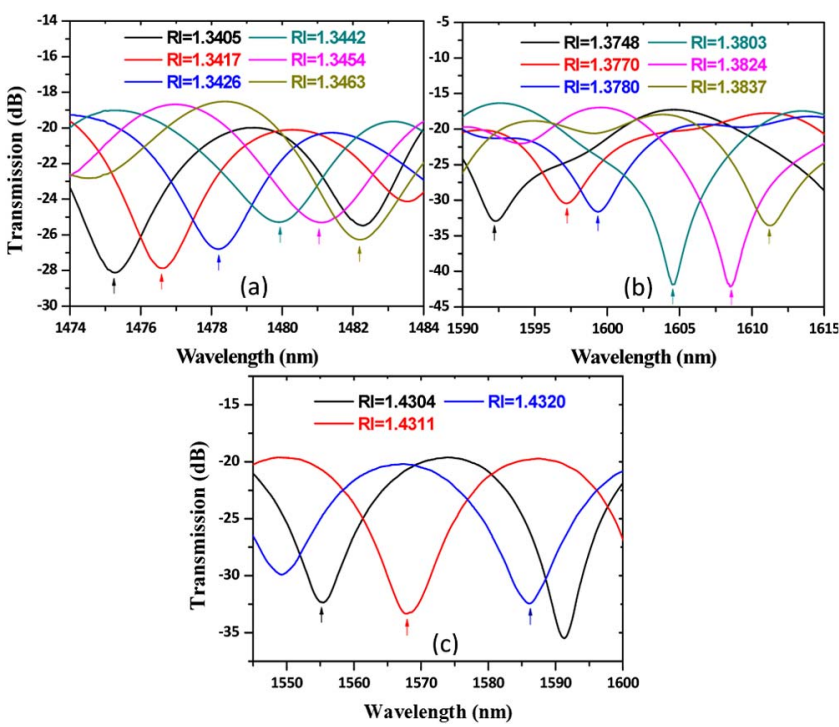

Fig. 2. Measured spectral responses of S-12.5 in different RI ranges: (a) $1.3405-1.3463$, (b) $1.3748-1.3837$, and (c) 1.4304-1.4320. 


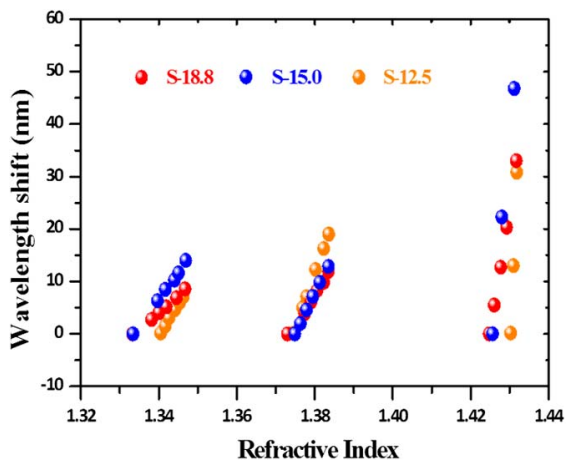

Fig. 3. Measured spectral wavelength shift versus RI for three different tapered SCSMF-structure-based sensors: S-18.8, S-15.0, and S-12.5.

in a higher RI liquid, which again also increases the portion of the evanescent wave exposed to the local environment.

As shown in Fig. 3 and Table 1, for all three samples, the linear correlative coefficients $R^{2}$ are greater than 0.992, which indicates that the wavelength shift exhibits good linear relationship with the RI change over a small RI range. The maximum sensitivities for S-18.8, S-15.0, and S-12.5 are $4722.9 \mathrm{~nm} / \mathrm{RIU} \quad(\mathrm{RI}=1.4249-1.4319), 8353.6 \mathrm{~nm} / \mathrm{RIU}$ $(\mathrm{RI}=1.4256-1.4312)$, and $19212.5 \mathrm{~nm} / \mathrm{RIU} \quad(\mathrm{RI}=$ $1.4304-1.4320)$, respectively. Taking into account that the OSA has a wavelength resolution of $0.01 \mathrm{~nm}$, the RI sensor based on S-12.5 has an RI resolution of $5.025 \times 10^{-7}$ which, to the best of our knowledge, is significantly higher than previously reported [16-20]. It is noted that, in this Letter, the central wavelength of the spectral dip is determined as the $3 \mathrm{~dB}$ mean wavelength, which is a more reliable parameter compared to the central peak wavelength.

Although the proposed sensor has very high RI sensitivity, this sensor suffers from the disadvantage of a narrow RI measurement range because of its limited free spectral range (typically $15 \mathrm{~nm}$, depending on the RI measurement range). One possible solution to overcome this problem is to combine a relatively low sensitivity RI sensor (for example, an SCSMF sensor without tapering) with the proposed tapered SCSMF. The lower sensitivity RI sensor would then be used to determine the approximate RI range of the analyte. Then the appropriate sensor would be applied to measuring a highly accurate RI value for the analyte.

It is noted that reducing the tapered waist diameter of such a fiber sensor might result in a higher RI sensitivity. However, a tapered fiber with a smaller waist diameter is more fragile compared to that of a larger waist diameter. Moreover, the tapered SCSMF sensor with a smaller waist diameter has a smaller free spectral range which results in a smaller RI measurement range. By considering the trade-off between the measurement range, sensitivity, and mechanical stability of the sensor, a minimum tapered waist diameter of $12.5 \mu \mathrm{m}$ was selected for our experiments.

Most of the evanescent RH sensors based on optical fibers reported to date require the coating of an additional humidity sensitive (or hygroscopic) material on the surface of the fiber sensor. Such humidity sensitive materials include polyvinyl alcohol (PVA), polyimide (PI), poly(methyl methacrylate) (PMMA), and nanoporous $\mathrm{TiO}_{2}$ and $\mathrm{SiO}_{2}$ films [21-23]. The underlying operating principle of these types of $\mathrm{RH}$ sensors is that the RI of the hygroscopic material coated on the surface of the sensor changes in response to changes in the humidity. The RI change results in a sensor spectral response variation, and, hence, the RH can be determined. However, sensors coated with humidity sensitive materials suffer from two main disadvantages: (1) the coating of the fiber with a humidity sensitive material requires an additional fabrication step which is difficult to control (usually because of the layer nonuniformity, etc.); and (2) the coating materials have limited lifetimes and are subject to contamination.

Dispensing with the need for a coating for an $\mathrm{RH}$ sensor is a very useful development, and, given the increase in RI sensitivity of the underlying sensor demonstrated already, here we explore whether it is possible to implement an RH sensor without the need for any additional humidity sensitive coatings on the fiber surface.

A schematic diagram of the RH testing system is shown in Fig. 4. In the experiment, an RI sensor with a tapered waist diameter of $12.5 \mu \mathrm{m}$ was placed in an RH chamber with normal air pressure (Electrotech Systems Inc., Model 5503-00 with Package F). Both the humidity and temperature can be controlled by using this system. The maximum available resolution of this $\mathrm{RH}$ control system is $0.1 \mathrm{RH} \%$, and all tests were carried out at a fixed temperature of $20.5 \pm 0.5^{\circ} \mathrm{C}$.

As shown above, sample S-12.5 has the highest RI sensitivity, and, hence, it was selected for this RH sensing demonstration. In our experiments, the $\mathrm{RH}$ within the chamber was increased gradually from $60 \%$ to $95 \%$ RH. Figure 5 (a) shows the spectral response of the sensor at different $\mathrm{RH}$ values. It is observed that, as the $\mathrm{RH}$ increases from $60 \%$ to $95 \%$, the central wavelength of the spectral dip shifts to a longer wavelength monotonically. The wavelength shift versus RH change is plotted in Fig. 5(b). It is clear that the wavelength shift changes exponentially with the increase of $\mathrm{RH}$ in the $\mathrm{RH}$ range, from $60.4 \%$ to $94.5 \%$. The total wavelength shift from $60.4 \%$ to $94.5 \%$ is circa $2.0 \mathrm{~nm}$, and the maximum sensitivity of $18.3 \mathrm{~nm} / \mathrm{RHU}$ was achieved in the $\mathrm{RH}$ range of $90.4 \%$ to $94.5 \%$. The $\mathrm{RH}$ sensitivity achieved in this experiment is over four times higher than that of the previously reported $\mathrm{RH}$ sensor, based on a bare fiber structure without a hygroscopic coating layer [24].

As to the source of the RH-induced spectral shift, it is known that water vapor can be adsorbed on a silica surface

Table 1. Sensor Sensitivities and Linear Fit Correlative Coefficients for Different Tapered Waist Diameters

\begin{tabular}{|c|c|c|c|c|c|c|c|c|c|}
\hline Sensors & & $S-18.8$ & & & S-15.0 & & & $S-12.5$ & \\
\hline RI Range & $\begin{array}{c}1.3333- \\
1.3468\end{array}$ & $\begin{array}{c}1.3775- \\
1.3836\end{array}$ & $\begin{array}{l}1.4249- \\
1.4319\end{array}$ & $\begin{array}{c}1.3334- \\
1.3469\end{array}$ & $\begin{array}{c}1.3749- \\
1.3835\end{array}$ & $\begin{array}{c}1.4256- \\
1.4312\end{array}$ & $\begin{array}{c}1.3405- \\
1.3463\end{array}$ & $\begin{array}{c}1.3748- \\
1.3837\end{array}$ & $\begin{array}{c}1.4304- \\
1.4320\end{array}$ \\
\hline $\begin{array}{l}\text { Linear Fit Correlative } \\
\text { Coefficient }\end{array}$ & 0.9956 & 0.9924 & 0.9968 & 0.9958 & 0.9989 & 0.9940 & 0.9949 & 0.9992 & 0.9990 \\
\hline Sensitivity (nm/RIU) & 634.8 & 1313.1 & 4722.9 & 1038.5 & 1496.5 & 8353.6 & 1198.3 & 2123.6 & 19212.5 \\
\hline
\end{tabular}




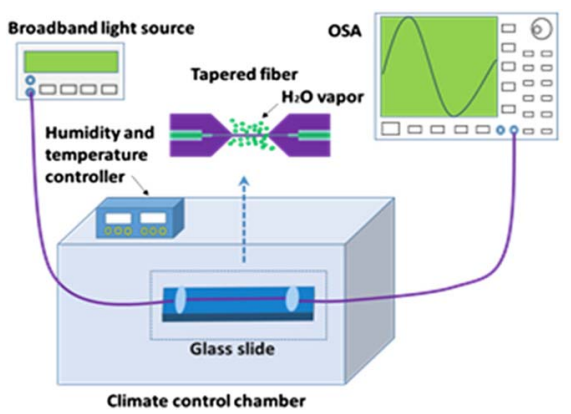

Fig. 4. Schematic diagram of the setup for the RH sensing experiment.
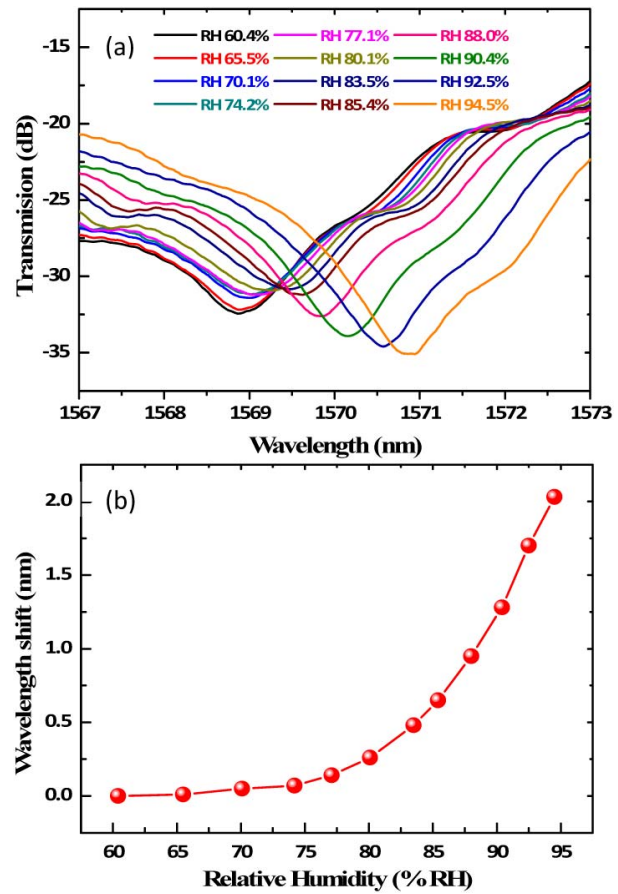

Fig. 5. (a) Measured spectral responses at different RH values for $\mathrm{S}$ 12.5, (b) wavelength shift versus RH.

and that this, in turn, will change the local RI. With an increase of the RH, more water vapor is adsorbed on the fiber surface, which increases the effective RI. In the simple demonstration carried out here, a further contributing factor to the spectral shift is that, since the tapered SCSMF is suspended slightly above the glass slide, an increase in the adsorbed water vapor can increase stress and introduce a bend to the tapered SCSMF section because of increased weight; this alters light propagation within the fiber which, in turn, leads to an additional source of spectral shift.

In conclusion, three tapered SCSMF fiber structures with different waist diameters $(12.5,15.0$, and $18.8 \mu \mathrm{m})$ were fabricated by using the microheater brushing technique. These structures were used for RI sensing. Experimental results show that all the samples have very high RI sensitivity and that the RI sensor with a tapered waist diameter of $12.5 \mu \mathrm{m}$ gives the highest sensitivity of $19212.5 \mathrm{~nm} / \mathrm{RIU}$ in the RI range of 1.4304 to 1.4320 . All sensors have a linear wavelength shift response versus RI changes. The sample with a tapered waist diameter of $12.5 \mu \mathrm{m}$ was used to test its $\mathrm{RH}$ sensing properties without any additional coating on the surface of the fiber. Experimental results indicate that the wavelength shift changes exponentially with the increase of $\mathrm{RH}$ in the $\mathrm{RH}$ range of $60 \%$ to $95 \% \mathrm{RH}$. A maximum RH sensitivity of $18.3 \mathrm{~nm} / \mathrm{RHU}$ was achieved in the $\mathrm{RH}$ range of $90.4 \%$ to $94.5 \%$.

Funding. FIOSRAIGH 2012 (Dean of Graduate Students' Award); Open Fund of IPOC (BUPT); Science Foundation Ireland (SFI) (SFI/13/ISCA/2845, SFI/13/TIDA/B2707).

\section{REFERENCES}

1. J. Wo, G. Wang, Y. Cui, Q. Sun, R. Liang, P. P. Shum, and D. Liu, Opt. Lett. 37, 67 (2012).

2. J. Villatoro and D. Monzón-Hernández, J. Lightwave Technol. 24, 1409 (2006).

3. W. Liang, Y. Huang, Y. Xu, R. K. Lee, and A. Yariv, Appl. Phys. Lett. 86, 151122 (2005).

4. J. Homola, S. S. Yee, and G. Gauglitz, Sens. Actuators, B 54, 3 (1999).

5. Q. Wu, Y. Semenova, P. Wang, and G. Farrell, Opt. Express 19, 7937 (2011).

6. V. Zamora, A. Díez, M. V. Andrés, and B. Gimeno, Opt. Express 15, 12011 (2007).

7. L. Bo, P. Wang, Y. Semenova, and G. Farrell, IEEE Photon. Technol. Lett. 25, 228 (2013)

8. Q. Wu, Y. Semenova, P. Wang, and G. Farrell, J. Opt. 13, 125401 (2011).

9. Q. Wu, Y. Semenova, J. Mathew, P. Wang, and G. Farrell, Opt. Lett. 36, 1752 (2011).

10. G. Brambilla, F. Xu, P. Horak, Y. Jung, F. Koizumi, N. P. Sessions, E. Koukharenko, X. Feng, G. S. Murugan, J. S. Wilkinson, and D. J. Richardson, Adv. Opt. Photon. 1, 107 (2009).

11. T. K. Yadav, R. Narayanaswamy, M. H. Abu Bakar, Y. M. Kamil, and M. A. Mahdi, Opt. Express 22, 22802 (2014).

12. S. Zhu, F. Pang, S. Huang, F. Zou, Y. Dong, and T. Wang, Opt. Express 23, 13880 (2015).

13. Z. Xu, Q. Sun, B. Li, Y. Luo, W. Lu, D. Liu, P. Shum, and L. Zhang, Opt. Express 23, 6662 (2015).

14. Q. Wu, Y. Ma, Y. Semenova, J. Yuan, L. Bo, P. Wang, X. Sang, M. Teng, C. Yu, C. C. O'Mahony, and G. Farrell, Proc. SPIE 9157, 91574U (2014).

15. G. Brambilla, V. Finazzi, and D. Richardson, Opt. Express 12, 2258 (2004).

16. Z. Tian, S. S.-H. Yam, and H.-P. Loock, IEEE Photon. Technol. Lett. 20, 1387 (2008)

17. A. Iadicicco, A. Cusano, A. Cutolo, R. Bernini, and M. Giordano, IEEE Photon. Technol. Lett. 16, 1149 (2004).

18. Z. Tian, S. S.-H. Yam, and H. P. Loock, Opt. Lett. 33, 1105 (2008).

19. Y. Wang, D. N. Wang, M. Yang, W. Hong, and P. Lu, Opt. Lett. 34, 3328 (2009).

20. C. Guan, X. Tian, S. Li, X. Zhong, J. Shi, and L. Yuan, Sens. Actuators, B 188, 768 (2013).

21. A. Gaston, F. Perez, and J. Sevilla, Appl. Opt. 43, 4127 (2004).

22. L. Alwis, T. Sun, and K. V. Grattan, IEEE Sensors J. 13, 767 (2013).

23. W. Zhang and D. J. Webb, Opt. Lett. 39, 3026 (2014).

24. M. Shao, X. Qiao, H. Fu, N. Zhao, Q. Liu, and H. Gao, IEEE Sensors J. 13, 2026 (2013) 